\title{
Ganglioneuroma of the Adrenal Gland: A Rare Tumor in a Rare Location
}

\author{
Hayim Gilshtein $^{\mathrm{a}} \quad$ Zvi Peled $^{\mathrm{a}} \quad$ Shahar Grunner $^{\mathrm{a}}$ \\ Doron Fischer $^{\mathrm{b}} \quad$ Eli Kakiashvili $^{\mathrm{a}}$ Yoram Kluger $^{\mathrm{a}}$ \\ ${ }^{a}$ Division of Surgical Oncology, Department of General Surgery, and ${ }^{b}$ Radiology \\ Department, Rambam Health Care Center, Haifa, Israel
}

\section{Key Words}

Ganglioneuroma $\cdot$ Adrenal $\cdot$ Sympathetic chain $\cdot$ Retroperitoneum $\cdot$ Neuroblastoma

\begin{abstract}
A 62-year-old man presented to his general practitioner complaining of non-specific back pain. He underwent a computerized tomography scan and magnetic resonance imaging that revealed a large left adrenal mass. A thorough investigation of this mass revealed it to be a non-secreting tumor. At surgery, a large tumor of the left adrenal was found. The final pathology report revealed a ganglioneuroma of the adrenal gland.
\end{abstract}

\section{Introduction}

Ganglioneuromas are benign and well-differentiated tumors of the sympathetic nervous system. They arise from the great sympathetic chains extending from the base of the skull to the neck, mediastinum, retroperitoneum and adrenal glands $[1,2]$. Ganglioneuromas of the adrenal gland are extremely rare. These tumors affect preferentially young people with the majority reported to occur before the age of 20 . These tumors are usually asymptomatic and are hormonally silent. When symptomatic, they present with non-specific symptoms related to their size or location with compression of neighboring structures [3].

Since adrenal ganglioneuromas are extremely rare, they are usually found through routine work-up for unrelated conditions [1]. Their most significant differential diagnosis is neuroblastoma tumors. Histopathological examination is currently the only tool to definitely diagnose this pathology. In the era of frequent use of CT scans for diagnosis as well as the use of laparoscopy in the management of incidental adrenal findings, awareness for this pathology is warranted. 
We herein describe a rare case of adrenal ganglioneuroma which was treated in our department. This entity is scarcely reported in the English literature.

\section{Case Report}

A 61-year-old male presented with complaints of lower back pain. His previous history consisted of ischemic heart disease with previous coronary bypass surgery, hypertension and hyperlipidemia. He had suffered from backache for several months, without fever, weight loss or other gastrointestinal-related symptoms. On physical examination there were no noticeable findings except for mild tenderness in his left flank. He underwent routine lab work, which was unremarkable. Due to the persistence of his symptoms he underwent a CT scan. An 8-cm non-homogenous mass was revealed in the left adrenal gland. An MRI was performed in order to further investigate the mass (fig. 1). On MRI an isointense left adrenal mass of $8.3 \times 4.4 \mathrm{~cm}$ was observed on T1. The mass was hyperintense on $\mathrm{T} 2$ and underwent non-homogenous enhancement during the portal phase with gadolinium. Endocrine work-up including urine catecholamine and cortisol levels was normal. Due to the tumor size and with consideration of the differential diagnosis of a malignant lesion we elected to operate on the patient. At laparotomy a large, $4 \times 6 \times 8 \mathrm{~cm}$ lesion of the left adrenal adherent to the diaphragm was found. The lesion was completely excised. The final histopathologic report revealed adrenal ganglioneuroma. Postoperative recovery was uneventful.

\section{Discussion}

Ganglioneuromas are benign tumors originating from neural crest cells and arising from great sympathetic chains. These tumors extend from the skull base to the retroperitoneum and rarely arise in the adrenal glands. They usually present in the younger age group, are discovered incidentally or due to non-specific symptoms caused by their mass effect on adjacent organs [3].

There are several features suggesting a ganglioneuroma in case of an adrenal tumor [4]: lack of hormonal secretion, evidence of calcifications, no vessel involvement and late enhancement on dynamic MRI with low T1 signal. High levels of urinary catecholamines as well as higher uptake of MIBG help to differentiate neuroblastoma from ganglioneuroma [5]. Although a high index of suspicion can aid in the diagnosis based on radiology findings, surgery is recommended as only tissue diagnosis can distinguish between the various adrenal pathologies.

Complete surgical excision reaching negative margins is the treatment of choice. The surgical approach can be either open or laparoscopic dependent on the tumor size and its differential diagnosis based on radiologic features. Complete removal of the tumor results in excellent outcome [1].

\section{Conclusion}

Ganglioneuroma of the adrenal gland is an extremely rare entity and as such represents a diagnostic challenge. The wide implementation of imaging modalities such as CT and MRI may result in increased diagnosis of such tumors. Thus, a high index of suspicion is needed to include this entity in the differential diagnosis of adrenal mass. Surgical excision with histopathology confirmation of the tumor pathology remains the mainstay of treatment and diagnosis. 


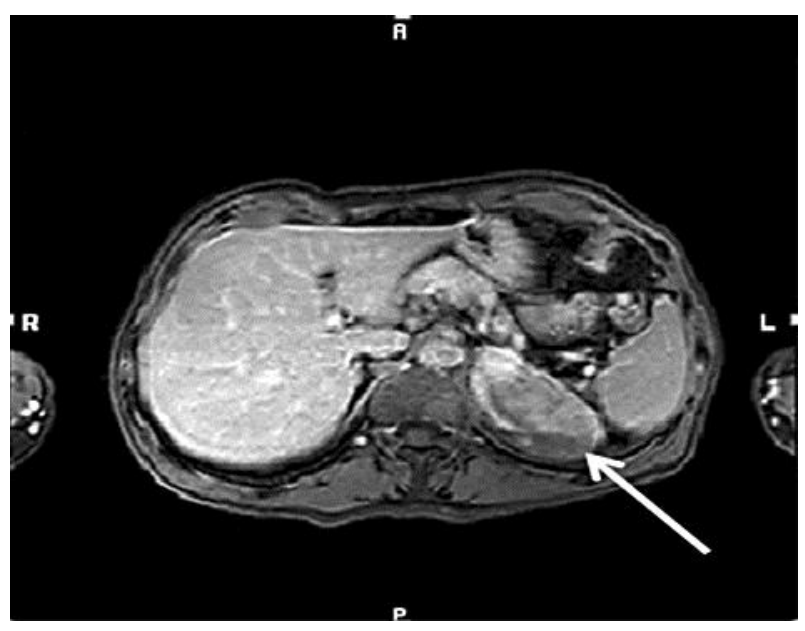

Fig. 1. A T2 MRI with the arrowhead pointing at a left adrenal mass.

\section{References}

1 Linos D, Tsirlis T, Kapralou A, et al: Adrenal ganglioneuromas: incidentalomas with misleading clinical and imaging features. Surgery 2011;149:99-105.

-2 Sucandy I, Akmal YM, Sheldon DG: Ganglioneuroma of the adrenal gland and retroperitoneum: a case report. N Am J Med Sci 2011;3:336-338.

-3 Hayes FA, Green AA, Rao BN: Clinical manifestation of ganglioneuroma. Cancer 1989;63:1211-1214.

-4 Maweja S, Materne R, Detrembleur N, et al: Adrenal ganglioneuroma. Am J Surg 2007;194:683-684.

5 Georger B, Hero B, Harms D, Grebe J, Scheidhauer K, Berthold F: Metabolic activity and clinical features of primary ganglioneuroma. Cancer 2001;91:1905-1913. 